\title{
Modified Schur-Cohn Criterion for Stability of Delayed Systems
}

\author{
Juan Ignacio Mulero-Martínez \\ Departamento de Ingeniería de Sistemas y Automática, Universidad Politécnica de Cartagena, \\ Campus Muralla del Mar, 30203 Cartagena, Spain \\ Correspondence should be addressed to Juan Ignacio Mulero-Martínez; juan.mulero@upct.es
}

Received 5 June 2014; Revised 11 September 2014; Accepted 14 September 2014

Academic Editor: Hak-Keung Lam

Copyright (C) 2015 Juan Ignacio Mulero-Martínez. This is an open access article distributed under the Creative Commons Attribution License, which permits unrestricted use, distribution, and reproduction in any medium, provided the original work is properly cited.

\begin{abstract}
A modified Schur-Cohn criterion for time-delay linear time-invariant systems is derived. The classical Schur-Cohn criterion has two main drawbacks; namely, (i) the dimension of the Schur-Cohn matrix generates some round-off errors eventually resulting in a polynomial of $s$ with erroneous coefficients and (ii) imaginary roots are very hard to detect when numerical errors creep in. In contrast to the classical Schur-Cohn criterion an alternative approach is proposed in this paper which is based on the application of triangular matrices over a polynomial ring in a similar way as in the Jury test of stability for discrete systems. The advantages of the proposed approach are that it halves the dimension of the polynomial and it only requires seeking real roots, making this modified criterion comparable to the Rekasius substitution criterion.
\end{abstract}

\section{Introduction}

Stability theory for linear time invariant systems with delays have long been in existence, but it still remains an active area of research, with applications in power system control, biology, human-in-the-loop control, neural networks, control in the context of automotive engines, haptic control, and synthetic biology, [1].

In this work we treat with linear time invariant timedelayed systems (LTI-TDS) with commensurate delays described by the state-space equation [2]:

$$
\dot{x}(t)=A_{0} x(t)+\sum_{k=1}^{m} A_{k} x(t-k \tau), \quad m \in \mathbb{Z}^{+},
$$

where $x \in \mathbb{R}^{n}$ stands for the state vector, $A_{0}, A_{k} \in \mathbb{R}^{n \times n}$ are given system matrices, and $\tau>0$ is a delay time. For these systems the characteristic quasi-polynomial $p_{c}(s)$ possesses the form of

$$
p_{c}(s, \tau)=\sum_{k=0}^{q} a_{k}(s) e^{-k \tau s},
$$

where $q$ is the degree of commensuracy in the dynamics and $a_{k} \in \mathbb{R}[s]$ are polynomials of degree at most $n-k$.
It is well known that the analysis of the stability of LTITDS lies on the root continuity argument; that is, the location of the poles of $p_{c}$ varies continuously with respect to delay. This means that any root crossing from the left half plane to the right half-plane will need to pass through the imaginary axis; as a result the computation of crossing frequencies is crucial when analyzing the stability and also for the plot of the root locus of $p_{c}$ as $\tau$ varies [3-9].

There are five criteria in the literature to compute delay margins of general time-delayed systems: (i) Schur-Cohn (Hermite matrix formulation) [2, 10-12]; (ii) elimination of transcendental terms in the characteristic equation [13]; (iii) matrix pencil, Kronecker sum method [2, 11, 12, 14]; (iv) Kronecker multiplication and elementary transformation [15]; (v) Rekasius substitution, [4, 16, 17]. These criteria are based on the determination of all the imaginary roots of the characteristic equation and require numerical procedures which result in vast range of precisions in finding pure imaginary roots of polynomials. Some criteria show subtle inapplicabilities mainly from a numerical implementation viewpoint [18]. In particular methods (i), (iii), and (iv) lead to polynomials of degree $2 q^{2}$, while (ii) requires finding the roots of a polynomial of degree $q 2^{q}$. The Kronecker multiplication method generates the results with the smallest 
number of significant digits among the above five methods. In the literature, the Rekasius substitution approach is the most attractive since the generated polynomial has degree $q^{2}$, much smaller than all the other criteria. Another advantage of this method is that only real roots are searched for, instead of complex ones, which is very crucial from a practical view point since complex roots are difficult to compute when numerical errors are dragged and propagated. For a total comparison of these methods, demonstrating their strengths and weakness, the reader is referred to [19].

This paper is focused on improving the Schur-Cohn procedure so as to obtain the advantageous properties of the Rekasius substitution criterion. Specifically, we modify the Schur-Cohn criterion to generate a real polynomial of degree $q^{2}$ whose real roots determine the pure imaginary roots of the characteristic equation. The original Schur-Cohn procedure computes the determinant of a partitioned matrix

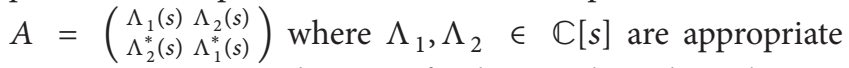
$q \times q$ matrices over the ring of polynomials in the unknown $s$ and $\Lambda^{*}$ denotes the Hermitian (or conjugated complex) of $\Lambda$. The classical Schur-Cohn procedure for high dimensions $\left(2 q^{2}>10\right)$ becomes numerically unreliable due to repeated round-off errors in the determinant expansion procedure unless the operation is performed using very large number of significant digits. This particular point alone brings a weakness from the numerical deployment viewpoint, since the operation ultimately yields poor precision in determining the desired imaginary roots: because of the accumulated numerical errors the imaginary roots shape up in the form of $s=j \omega+\epsilon, \epsilon \ll 1$ and $j=\sqrt{-1}$, which leads the numerical error in real parts to be at least in the order of 10 magnitudes larger than the computational precision. Therefore, it is problematic to decide whether these roots, which are very close to the imaginary axis, are really imaginary roots or not. As an attempt to overcome the problems of the SchurCohn method some authors have proposed a modification based on the determination of the real eigenvalues of a constant matrix, [11]. By contrast, our modification stems from the applications of some transformations on $A$ by using triangular matrices over the polynomial ring in a similar way as in the Jury test of stability for discrete systems [20].

The remainder of the paper is structured as follows: in Section 2, the basic idea of the modified stability criterion is explained and a numerical example is provided. Finally, a summary and an outlook are given in Section 3.

\section{Delayed Systems. Schur-Cohn Modified Method}

The stability of the system (1) is determined by its characteristic quasi-polynomial in $s$ [21]:

$$
\begin{aligned}
p_{c}\left(s ; e^{-s \tau}, \ldots, e^{-m s \tau}\right) & =\operatorname{det}\left(s I-A_{0}-\sum_{k=1}^{m} A_{k} e^{-s k \tau}\right) \\
& =\sum_{k=0}^{q} a_{k}(s) e^{-k \tau s},
\end{aligned}
$$

$$
\begin{gathered}
a_{0}(s)=s^{n}+\sum_{i=0}^{n-1} a_{0 i} s^{i}, \\
a_{k}(s)=\sum_{i=0}^{n-1} a_{k i} s^{i}, \quad k=1, \ldots, q .
\end{gathered}
$$

The characteristic quasi-polynomial contains time delays of commensurate nature with degree $q$; that is, $p_{c}$ depends on $e^{-k \tau s}$ for $k=0, \ldots, q$, with $a_{q}(s) \neq 0$ and $\operatorname{rank}\left(A_{k}\right) \leq q \leq$ $n=\operatorname{rank}\left(A_{0}\right)$. The starting-point of all the stability criteria is the determination of the roots of $p_{c}$ on the imaginary axis. In fact, it is well known from the stability theory of LTITDS that the pure imaginary characteristic roots are the only possible transition points from stable to unstable behavior, and vice versa. We want to determine all the imaginary roots of $p_{c}\left(s ; e^{-s \tau}, \ldots, e^{-m s \tau}\right)$, and to this end it is convenient to introduce the variable $z=e^{-s \tau}$ and write $p_{c}$ as a polynomial in two unknowns over the field of reals, $p_{c}(s, z)=\sum_{i=0}^{q} a_{k}(s) z^{k} \in$ $\mathbb{R}[s, z]$.

We define the bivariate polynomial $r \in \mathbb{C}[\omega, z]$ as $r(\omega, z)=\sum_{i=0}^{q} b_{i}(\omega) z^{i}$ such that $b_{i}(\omega)=a_{i}(j \omega) \in \mathbb{C}[\omega]$. With every bivariate polynomial over the complex field, $r(\omega, z)$, we associate two triangular matrices over the polynomial ring $\mathbb{C}[\omega]:$

$$
\begin{aligned}
& U_{q}(r(\omega)) \\
& =\left(\begin{array}{ccccc}
\bar{b}_{q}(\omega) & \bar{b}_{q-1}(\omega) & \bar{b}_{q-2}(\omega) & \ldots & \bar{b}_{1}(\omega) \\
0 & \bar{b}_{q}(\omega) & \bar{b}_{q-1}(\omega) & \ldots & \bar{b}_{2}(\omega) \\
\vdots & & & & \vdots \\
0 & \ldots & 0 & \bar{b}_{q}(\omega) & \bar{b}_{q-1}(\omega) \\
0 & \ldots & 0 & 0 & \bar{b}_{q}(\omega)
\end{array}\right), \\
& L_{q}(r(\omega)) \\
& =\left(\begin{array}{ccccc}
0 & \cdots & 0 & 0 & b_{0}(\omega) \\
0 & \cdots & 0 & b_{0}(\omega) & b_{1}(\omega) \\
\vdots & & & & \\
0 & b_{0}(\omega) & b_{1}(\omega) & \cdots & b_{q-2}(\omega) \\
b_{0}(\omega) & b_{1}(\omega) & b_{2}(\omega) & \cdots & b_{q-1}(\omega)
\end{array}\right) .
\end{aligned}
$$

For the sake of simplicity we write $U_{q}, L_{q}$ instead of $U_{q}(r(\omega)), L_{q}(r(\omega))$. The Schur-Cohn criterion solves the problem of computing the values of $\omega$ such that $p_{c}\left(j \omega ; z, \ldots, z^{q}\right)=0$ by multiplying $p_{c}\left(j \omega ; z, \ldots, z^{q}\right)$ by $z^{-k}$ for $k=0, \ldots, q-1$, and $\overline{p_{c}\left(j \omega ; z, \ldots, z^{q}\right)}$ by $z^{k}$ for $k=1, \ldots, q$. This leads to a system of $2 q$ homogeneous equations in the unknowns $z^{k}, k=-q, \ldots, 0, \ldots, q-1$ which has the following matrix representation:

$$
\left(\begin{array}{cc}
L_{q} & \bar{U}_{q} J \\
J U_{q} & J \bar{L}_{q} J
\end{array}\right)\left(\begin{array}{l}
\mathbf{e}_{1} \\
\mathbf{e}_{2}
\end{array}\right)=\left(\begin{array}{l}
0 \\
0
\end{array}\right)
$$

where $J$ is the counteridentity matrix (i.e. an antidiagonal matrix with ones), $\mathbf{e}_{1}=J l_{q-1}^{T}(z), \mathbf{e}_{2}=J l_{q-1}^{T}\left(z^{-1}\right) z^{-1}$, and 
$l_{q-1}^{T}(z)=\left(1, z, z^{2}, \ldots, z^{q-1}\right) ;$ the homogeneous system in (7) is the starting point of the Schur-Cohn method. If we make the change of unknowns $\mathbf{e}_{i} \mapsto J \mathbf{e}_{i}$ and we take into account the idempotence of $J,(6)$ can be transformed into

$$
\left(\begin{array}{cc}
L_{q} & \bar{U}_{q} \\
J U_{q} & J \bar{L}_{q}
\end{array}\right)\left(\begin{array}{l}
J \mathbf{e}_{1} \\
J \mathbf{e}_{2}
\end{array}\right)=\left(\begin{array}{l}
0 \\
0
\end{array}\right) .
$$

It is easily verified that $U_{q}^{*} \bar{L}_{q}$ is of the same structure as $L_{q}$, and in particular it is symmetric. Therefore $U_{q}^{*} \bar{L}_{q}=\left(U_{q}^{*} \bar{L}_{q}\right)^{T}=$ $L_{q}^{*} \bar{U}_{q}$. As a consequence we obtain

$$
\begin{aligned}
& \left(\begin{array}{cc}
L_{q}^{*} & -U_{q}^{*} \\
O & I
\end{array}\right)\left(\begin{array}{cc}
I & O \\
O & J
\end{array}\right)\left(\begin{array}{cc}
L_{q} & \bar{U}_{q} \\
J U_{q} & J \bar{L}_{q}
\end{array}\right) \\
& =\left(\begin{array}{cc}
L_{q}^{*} & -U_{q}^{*} \\
O & I
\end{array}\right)\left(\begin{array}{cc}
L_{q} & \bar{U}_{q} \\
U_{q} & \bar{L}_{q}
\end{array}\right) \\
& =\left(\begin{array}{cc}
L_{q}^{*} L_{q}-U_{q}^{*} U_{q} & O \\
U_{q} & \bar{L}_{q}
\end{array}\right) .
\end{aligned}
$$

The homogeneous system (7) has a nontrivial solution so long as $\operatorname{det}\left(\begin{array}{cc}L_{q} & \bar{U}_{q} \\ J U_{q} & J \bar{L}_{q}\end{array}\right)=0$. We use the fact that

$$
\begin{aligned}
& \operatorname{det}\left(\left(\begin{array}{cc}
L_{q}^{*} & -U_{q}^{*} \\
O & I
\end{array}\right)\left(\begin{array}{cc}
I & O \\
O & J
\end{array}\right)\right) \\
& =\operatorname{det}\left(\begin{array}{cc}
L_{q}^{*} & -U_{q}^{*} J \\
O & J
\end{array}\right)=\operatorname{det}\left(L_{q}^{*} J\right)=\operatorname{det}\left(\bar{L}_{q}\right)(-1)^{q},
\end{aligned}
$$

and taking determinants in (8) yields

$$
\begin{gathered}
\operatorname{det}\left(\bar{L}_{q}\right)(-1)^{q} \operatorname{det}\left(\begin{array}{cc}
L_{q} & \bar{U}_{q} \\
J U_{q} & J \bar{L}_{q}
\end{array}\right) \\
=\operatorname{det}\left(\bar{L}_{q}\right) \operatorname{det}\left(L_{q}^{*} L_{q}-U_{q}^{*} U_{q}\right) .
\end{gathered}
$$

As a result,

$$
\operatorname{det}\left(\begin{array}{cc}
L_{q} & \bar{U}_{q} \\
J U_{q} & J \bar{L}_{q}
\end{array}\right)=(-1)^{q} \operatorname{det}\left(L_{q}^{*} L_{q}-U_{q}^{*} U_{q}\right) .
$$

Therefore, the problem has been reduced to find the pure imaginary roots of $\operatorname{det}\left(L_{q}^{*} L_{q}-U_{q}^{*} U_{q}\right)$ which is just the determinant of the Jury matrix $J_{q}(r(\omega))$ over $\mathbb{C}[\omega]$ defined as

$$
J_{q}(r(\omega))=\left(\begin{array}{ll}
L_{q} & U_{q} \\
\bar{U}_{q} & \bar{L}_{q}
\end{array}\right) \in \mathbb{C}^{2 q \times 2 q}[\omega] .
$$

This can be seen by a similar argument as above in (8),

$$
\begin{aligned}
& \left(\begin{array}{cc}
L_{q}^{*} & -U_{q}^{*} \\
O & I
\end{array}\right)\left(\begin{array}{cc}
L_{q} & \bar{U}_{q} \\
U_{q} & \bar{L}_{q}
\end{array}\right) \\
& =\left(\begin{array}{cc}
L_{q}^{*} L_{q}-U_{q}^{*} U_{q} & L_{q}^{*} \bar{U}_{q}-U_{q}^{*} \bar{L}_{q} \\
U_{q} & \bar{L}_{q}
\end{array}\right) \\
& =\left(\begin{array}{cc}
L_{q}^{*} L_{q}-U_{q}^{*} U_{q} & O \\
U_{q} & \bar{L}_{q}
\end{array}\right),
\end{aligned}
$$

and $\operatorname{det}\left(J_{q}(r(\omega))\right)=\operatorname{det}\left(L_{q}^{*} L_{q}-U_{q}^{*} U_{q}\right)$.
In the sequel we define the polynomial $\zeta(\omega)$ := $\operatorname{det}\left(J_{q}(r(\omega))\right)$ and the set of zeros of a polynomial $p$ is denoted as $\mathscr{Z}(p)$.

Proposition 1. The real polynomial $\zeta(\omega)$ is even.

Proof. We prove that $\zeta(\lambda)=0$ if and only if $\zeta(-\lambda)=0$. First of all note that $b_{i}(-\omega)=\overline{b_{i}(\omega)}$ and, then, $r(-\omega, z)=\bar{r}(\omega, z)$. As a consequence,

$$
\begin{aligned}
& U_{q}(\bar{r}(\omega))=\bar{U}_{q}(r(\omega))=U_{q}(r(-\omega)), \\
& L_{q}(\bar{r}(\omega))=\bar{L}_{q}(r(\omega))=L_{q}(r(-\omega)) .
\end{aligned}
$$

Thus,

$$
\begin{aligned}
& \qquad \begin{array}{l}
L_{q}^{*}(r(-\omega)) L_{q}(r(-\omega))-U_{q}^{*}(r(-\omega)) U_{q}(r(-\omega)) \\
\quad=L_{q}^{T}(r(\omega)) \bar{L}_{q}(r(\omega))-U_{q}^{T}(r(\omega)) \bar{U}_{q}(r(\omega)), \\
\text { and } \zeta(\lambda)=\zeta(-\lambda) .
\end{array}
\end{aligned}
$$

From the above development we obtain the following criterion.

Criterion 1. Let $\omega_{0} \in \mathbb{R}^{+}$be a real root of $\zeta(\sqrt{s})$, then $\pm j \sqrt{\omega_{0}}$ is a pair of pure imaginary roots of the LTI-TDS system in (1).

Proof. It is immediate that $\pm j \omega$ is a pair of pure imaginary roots of $p_{c}\left(s ; z, \ldots, z^{q}\right)$ if and only if $\omega$ is a positive real root of $p_{c}\left(j \omega ; z, \ldots, z^{q}\right)$. According to the Schur-Cohn method the roots of $p_{c}\left(j \omega ; z, \ldots, z^{q}\right)$ can be determined by searching for the real roots in the system (7). Additionally, it was shown above that the solution of (7) implies the computing of the roots of the even polynomial $\zeta(\omega)$; henceforth, if $\omega_{0} \in \mathbb{R}^{+}$is a root of $\zeta(\sqrt{s}), \pm \sqrt{\omega_{0}}$ is a pair of real roots of $\zeta(s)$ and $\pm j \omega_{0}$ is a pair of pure imaginary roots of $p_{c}\left(s ; z, \ldots, z^{q}\right)$.

Note that this criterion is advantageous since firstly we only need to compute real roots and secondly $\operatorname{deg}(\zeta(\sqrt{\cdot}))=q$. In the following the set of crossing frequencies is defined as

$$
\Omega:=\left\{\omega \in \mathbb{R}^{+}: \omega^{2} \in \mathscr{Z}(\zeta(\sqrt{\cdot})) \cap \mathbb{R}^{+}\right\} .
$$

Example 2. Let us consider the LTI-TDS system $\dot{x}(t)=$ $A_{0} x(t)+A_{1} x(t-\tau)$ where the system matrices are defined as

$$
A_{0}=\left(\begin{array}{ccc}
-1 & 13.5 & -1 \\
-3 & -1 & -2 \\
-2 & -1 & -4
\end{array}\right), \quad A_{1}=\left(\begin{array}{ccc}
-5.9 & 7.1 & -70.3 \\
2 & -1 & 5 \\
2 & 0 & 6
\end{array}\right)
$$

The quasi-polynomial characteristic is

$$
\begin{aligned}
& p_{c}\left(s ; e^{-s \tau}, \ldots, e^{-m s \tau}\right) \\
& \quad=a_{3}(s) e^{-3 \tau s}+a_{2}(s) e^{-2 \tau s}+a_{1}(s) e^{-\tau s}+a_{0}(s)
\end{aligned}
$$


with

$$
\begin{aligned}
& a_{0}(s)=s^{3}+6 s^{2}+45.5 s+111 \\
& a_{1}(s)=0.9 s^{2}-116.8 s-22.1 \\
& a_{2}(s)=90.9 s-185.1 \\
& a_{3}(s)=119.4
\end{aligned}
$$

In the frequency domain $(s=j \omega)$, we define the bivariate polynomial $r(\omega, z)=\sum_{i=0}^{3} a_{i}(j \omega) z^{i}$, and from (19) we build the triangular matrices $U_{3}(r)$ and $L_{3}(r)$ as follows:

$$
\begin{aligned}
U_{3}= & \left(\begin{array}{ccc}
119.4 & -185.1 & -0.9 \omega^{2}-22.1 \\
0 & 119.4 & -185.1 \\
0 & 0 & 119.4
\end{array}\right) \\
& +j \omega\left(\begin{array}{ccc}
0 & -90.9 & 116.8 \\
0 & 0 & -90.9 \\
0 & 0 & 0
\end{array}\right) \\
L_{3}= & \left(\begin{array}{ccc}
0 & 0 & 111.0-6.0 \omega^{2} \\
0 & 111.0-6.0 \omega^{2} & -0.9 \omega^{2}-22.1 \\
111.0-6.0 \omega^{2} & -0.9 \omega^{2}-22.1 & -185.1
\end{array}\right) \\
& +j \omega\left(\begin{array}{ccc}
0 & 0 & 45.5-\omega^{2} \\
0 & 45.5-\omega^{2} & -116.8 \\
45.5-\omega^{2} & -116.8 & 90.9
\end{array}\right) .
\end{aligned}
$$

Then we compute the polynomial $\zeta(\sqrt{s})$ according to Criterion 1 :

$$
\begin{aligned}
\zeta(\sqrt{s})= & s^{9}-165.81 s^{8}-18682 s^{7}+2.4869 \times 10^{5} s^{6} \\
& +35185 s^{5}-4.1197 \times 10^{9} s^{4}+9.4110 \times 10^{10} s^{3} \\
& -7.1137 \times 10^{11} s^{2}+1.8933 \times 10^{12} s \\
& -1.0144 \times 10^{12}
\end{aligned}
$$

whose positive real roots are $\mathscr{Z}(\zeta(\sqrt{\cdot})) \cap \mathbb{R}^{+}=$ $\{8.4802,4.4564,9.2141,0.70634,240.35\}$ and then

$$
\pm j \Omega=\{ \pm j 2.9121, \pm j 2.111, \pm j 3.0355, \pm j 0.84044, \pm j 15.503\}
$$

is the set of pure imaginary roots of the delayed system. Note that from the fundamental theorem of algebra there are only a finite number of crossing frequencies and that $\mathscr{Z}(\zeta(\sqrt{\cdot})) \cap \mathbb{R}^{+}$ represents the set of squares of crossing frequencies.

The method presented above is the first step to test the stability of the characteristic quasi-polynomial $p_{c}\left(s, e^{-s \tau}\right)$ and to compute the delay margin for LTI delay systems. It is common practice to make the following assumption on $p_{c}$.

Assumption 3. The characteristic quasi-polynomial $p_{c}$ is stable at $\tau=0$, that is, $\mathscr{Z}\left(p_{c}(\cdot ; 0)\right) \subset \mathbb{C}^{-}$, where $\mathbb{C}^{-}:=\{s \in \mathbb{C}$ : $\operatorname{Re}\{s\}<0\}$.
Recall that the delay margin $\bar{\tau}$ is the smallest deviation of $\tau$ from $\tau=0$ such that the system becomes unstable:

$$
\bar{\tau}:=\min \left\{\tau \geq 0: p_{c}\left(j \omega, e^{-j \omega \tau}\right)=0 \text { for some } \omega \in \mathbb{R}^{+}\right\} .
$$

For any $\tau \in[0, \bar{\tau})$, the system is stable, and whenever $\tau=\infty$, the system is said to be stable independent of delay. For each finite delay $\tau$ we can associate a crossing frequency which represents the first contact or crossing of the roots of $p_{c}$ from the stable region to the unstable one. The determination of the delay margin requires solving the bivariate polynomial $p_{c} \in$ $\mathbb{C}[\omega, z]$ in two phases: (i) computing the positive real roots of $\zeta(\sqrt{s})$ so as to obtain the crossing frequencies $\omega \in \Omega$ of $p_{c}$, (ii) finding the roots of $p_{c}(j \omega) \in \mathbb{C}[z]$ on the boundary of the unit disk $\mathbb{D}$ (denoted as $\partial \mathbb{D}$ ), at each crossing frequency $\omega$. A detailed analysis of the delay margin $\bar{\tau}$ can be found in [11] and in Theorem 2.11, pp. 59-60 in [2]. Under the assumption that $p_{c}$ is stable at $\tau=0$, the two-step stability method is summarized in Table 1.

Example 4. Taking into account the positive crossing frequencies $\omega_{k} \in \Omega$ computed for the LTI-TDS system in Example 2, we can derive the set of roots of $p_{c}\left(j \omega_{k}\right) \in \mathbb{C}[z]$ onto the unit circle $\partial \mathbb{D}$. Let $z_{k} \in \partial \mathbb{D} \cap \mathscr{E}\left(p_{c}\left(j \omega_{k}\right)\right)$, from the identity $z_{k}=e^{-j \omega_{k} \tau}=e^{j \arg \left(z_{k}\right)}$ it follows that $\tau_{k}=$ $\left(2 \pi-\arg \left(z_{k}\right)\right) / \omega_{k}$ (with $\left.\arg \left(z_{k}\right) \in[0,2 \pi]\right)$ and the system can cross the stability boundary $\partial \mathbb{C}=j \mathbb{R}$ from the stable halfplane $\mathbb{C}^{-}$to the unstable half-plane $\mathbb{C}^{+}:=\mathbb{C} \backslash\left(\mathbb{C}^{-} \cup \partial \mathbb{C}\right)$ or vice versa at $\tau_{k}$. If the root crosses $\partial \mathbb{C}$ from $\mathbb{C}^{+}$to $\mathbb{C}^{-}$at $\tau_{k}$, there exists a $\tau<\tau_{k}$ such that makes the root to cross from $\mathbb{C}^{-}$to $\mathbb{C}^{+}$. As a consequence the stability margin is defined as

$$
\bar{\tau}=\min _{1 \leq k \leq q}\left\{\frac{2 \pi-\arg \left(z_{k}\right)}{\omega_{k}}: \omega_{k} \in \Omega, z_{k} \in \partial \mathbb{D} \cap \mathscr{Z}\left(p_{c}\left(j \omega_{k}\right)\right)\right\} .
$$

Under Assumption 3 the system is unstable at $\tau=\bar{\tau}$ and at least a root crosses $\partial \mathbb{C}$ from $\mathbb{C}^{-}$to $\mathbb{C}^{+}$at $\bar{\tau}$. If we choose $\tau \in[0, \bar{\tau})$, then $\tau \omega_{k} \neq \theta_{k}$ and $p_{c}\left(j \omega_{k} ; e^{-j \omega \tau}\right) \neq 0$ for all $\omega \in \mathbb{R}^{+}$. Therefore, $p_{c}\left(s ; e^{-s \tau}\right)$ is stable for all $\tau \in[0, \bar{\tau})$. For each crossing frequency $\omega_{k}$ a delay $\tau_{k}$ is computed as shown in Table 2 . The delay margin is then computed as the minimum value of these delays, that is, $\bar{\tau}=\min \left\{\tau_{k}: k=1, \ldots, 5\right\}=$ 0.16230 .

The crossing direction of those roots onto the imaginary axis (either from stable to unstable complex plane or vice versa) as $\tau$ increases is a quantity called the root tendency (RT), $[7,8]$. For a cross frequency $\omega_{0}$ and a delay $\tau_{0}$, the root tendency is defined as

$$
(\mathrm{RT})_{\omega, \tau}=\operatorname{sgn}\left(\operatorname{Re}\left\{\left.\frac{\partial s}{\partial \tau}\right|_{\left(j \omega_{0}, \tau_{0}\right)}\right\}\right),
$$

where $\operatorname{sgn}(\cdot)$ denotes the sign function. In the following lemma we propose a simple method to compute the root tendency based on the implicit function theorem.

Lemma 5 (root tendency). Let $p_{c}$ be a characteristic quasipolynomial as defined in (3) and let $\tau_{0}$ be the delay for 
TABLE 1: Stability analysis of the quasipolynomial $p_{c}$ in terms of the delay margin $\bar{\tau}$.

\begin{tabular}{lll}
\hline Set of squares of crossing frequencies & Solutions of $p_{c}(\omega) \in \mathbb{C}[z]$ on $\partial \mathbb{D}, \omega \in \Omega$ & Stability of the quasi-polynomial $p_{c} \in \mathbb{C}[\omega, z]$ \\
\hline $\mathscr{Z}(\zeta(\sqrt{\cdot})) \cap \mathbb{R}^{+}=\varnothing$ & & \\
$\mathscr{Z}(\zeta(\sqrt{\cdot})) \cap \mathbb{R}^{+}=(0)$ & Stable, independent of delay $(\bar{\tau}=\infty)$ \\
$\mathscr{Z}(\zeta(\sqrt{ })) \cap \mathbb{R}^{+} \neq \varnothing, \mathscr{Z}(q) \cap \mathbb{R}^{+} \neq(0)$ & $\mathscr{Z}\left(p_{c}(j \omega)\right) \cap \partial \mathbb{D}=\varnothing$ & \\
& $\mathscr{E}\left(p_{c}(j \omega)\right) \cap \partial \mathbb{D} \neq \varnothing$ & Stable, dependent of delay $(\bar{\tau}<\infty)$ \\
\hline
\end{tabular}

TABLE 2: Delays corresponding to the crossing frequencies of the LTI-TDS system of the Example 2.

\begin{tabular}{lccc}
\hline Crossing frequency $\left(\omega_{k}\right)$ & Roots of $p_{c}\left(j \omega_{k}\right)$ on $\partial \mathbb{D}\left(z_{k}=e^{-j \omega_{k} \tau}\right)$ & Phase of $z_{k}\left(\theta_{k}\right)$ & Delay $\left(\tau_{k}\right)$ \\
\hline 2.9121 & $0.85692-j 0.51548$ & 5.7416 & 0.18597 \\
2.111 & $-0.26768-j 0.96352$ & 4.4414 & 0.87247 \\
3.0355 & $0.88110-j 0.47297$ & 5.7905 & 0.16230 \\
0.84044 & $0.97521+j 0.22129$ & 0.22314 & 7.2106 \\
15.503 & $-0.95537+j 0.29541$ & 2.8417 & 0.22199 \\
\hline
\end{tabular}

TABLE 3: Root tendency for the crossing frequencies of the LTI-TDS system of the Example 2.

\begin{tabular}{lccc}
\hline Crossing frequency $\left(\omega_{k}\right)$ & Delay $\left(\tau_{k}\right)$ & $\sum_{k=0}^{q}\left(a_{k}^{\prime}\left(j \omega_{0}\right)-(k-1) \tau_{0} a_{k}\left(j \omega_{0}\right)\right) e^{-j \omega_{0} k \tau_{0}}$ & $\sum_{k=1}^{q} k a_{k}\left(j \omega_{0}\right) e^{-j k \omega_{0} \tau_{0}}$ \\
\hline 2.9121 & 0.18597 & $-48.520+j 30.258$ & $74.136-j 58.701$ \\
2.111 & 0.87247 & $-140.62+j 342.86$ & $148.78-j 182.37$ \\
3.0355 & 0.16230 & $-47.511+j 27.405$ & $94.101-j 41.172$ \\
0.84044 & 7.2106 & $872.31-j 692.90$ & $-119.43+j 99.556$ \\
15.503 & 0.22199 & $-902.35-j 919.93$ & $1825.5+j 4475.4$ \\
\hline
\end{tabular}

which the system crosses the stability boundary at $j \omega_{0}$, for some $\omega_{0} \in \Omega$, that is, $p_{c}\left(j \omega_{0} ; \tau_{0}\right)=0$. Let us assume that $\left.\left(\partial p_{c}(s ; \tau) / \partial s\right)\right|_{\left(j \omega_{0}, \tau_{0}\right)}=\sum_{k=0}^{q}\left(a_{k}^{\prime}\left(j \omega_{0}\right)-(k-1) \tau a_{k}\left(\omega_{0}\right)\right)$ $e^{-j \omega_{0} k \tau_{0}} \neq 0$. Then, the root tendency of $j \omega_{0}$ is given by

$$
\begin{aligned}
& \text { ( RT })_{\omega_{0}, \tau_{0}} \\
& =-\operatorname{sgn}\left(\operatorname { I m } \left(\left(\sum_{k=1}^{q} k a_{k}\left(j \omega_{0}\right) e^{-j k \omega_{0} \tau_{0}}\right)\right.\right. \\
& \times\left(\sum_{k=0}^{q}\left(a_{k}^{\prime}\left(j \omega_{0}\right)-(k-1) \tau_{0} a_{k}\left(j \omega_{0}\right)\right)\right. \\
& \left.\left.\left.\times e^{-j k \omega_{0} \tau_{0}}\right)^{-1}\right)\right) \text {. }
\end{aligned}
$$

Proof. In virtue of the implicit function theorem, there exist a $\delta>0$, a neighborhood $U_{0} \subset \mathbb{C}$ of $j \omega_{0}$, and a continuous function $s:\left(\tau_{0}-\delta, \tau_{0}+\delta\right) \rightarrow U_{0}$ such that $p_{c}(s(\tau) ; \tau)=0$ for all $\tau \in\left(\tau_{0}-\delta, \tau_{0}+\delta\right)$; this is also obvious from the continuity of the roots $s(\cdot)$ with respect to the delay $\tau$. Furthermore, since $p_{c}$ is differentiable and $\left.\left(\partial p_{c}(s ; \tau) / \partial s\right)\right|_{\left(j \omega_{0}, \tau_{0}\right)} \neq 0$, it is clear that

$$
\left.\frac{\partial s}{\partial \tau}\right|_{\left(j \omega_{0}, \tau_{0}\right)}=-\frac{\partial p_{c}(s ; \tau) /\left.\partial \tau\right|_{\left(j \omega_{0}, \tau_{0}\right)}}{\partial p_{c}(s ; \tau) /\left.\partial s\right|_{\left(j \omega_{0}, \tau_{0}\right)}}
$$

where

$$
\begin{aligned}
& \left.\frac{\partial p_{c}(s ; \tau)}{\partial s}\right|_{\left(j \omega_{0}, \tau_{0}\right)} \\
& =\sum_{k=0}^{q}\left(a_{k}^{\prime}\left(j \omega_{0}\right)-(k-1) \tau_{0} a_{k}\left(j \omega_{0}\right)\right) e^{-j k \omega_{0} \tau_{0}} \\
& \quad+p_{c}\left(j \omega_{0} ; \tau_{0}\right), \\
& \left.\frac{\partial p_{c}(s ; \tau)}{\partial \tau}\right|_{\left(j \omega_{0}, \tau_{0}\right)}=-j \omega_{0} \sum_{k=1}^{q} k a_{k}\left(j \omega_{0}\right) e^{-j k \omega_{0} \tau_{0}} .
\end{aligned}
$$

Owing to $p_{c}\left(j \omega_{0} ; \tau_{0}\right)=0$ and from the chain rule of derivation, the result in (26) follows immediately.

Example 6. Following with Example 2 and using the root tendency lemma we can compute the crossing direction for each crossing frequency as shown in the fifth column in Table 3; RT $=-1$ indicates that the crossing is from $\mathbb{C}^{+}$to $\mathbb{C}^{-}$and $\mathrm{RT}=+1$ implies a crossing from $\mathbb{C}^{-}$to $\mathbb{C}^{+}$.

We finish this analysis by noting that it is not possible in general to separate $\zeta(\sqrt{s})$ with a factor containing exactly all the real roots of $\zeta(\sqrt{s})$ as stated in the following lemma.

Lemma 7. Given a polynomial $p \in \mathbb{R}_{n}[s]$ there does not exist a general procedure of factorization of $p$ in polynomials $p_{R} \in$ $\mathbb{R}[s]$ and $p_{C} \in \mathbb{R}[s], p(s)=p_{R}(s) p_{C}(s)$, such that $p_{R}$ includes exactly all the real roots of $p$ (and $p_{C}$ all the pairs of complex conjugated roots of $p$ not in $\mathbb{R})$. 
Proof. We prove this assertion by a counterexample: without loss of generality we assume that $p$ is monic. Let us assume that $p$ has only real roots which are ordered as $\alpha_{1}<\alpha_{2}<$ $\cdots<\alpha_{m}, m \leq n$. Always we can find a value $\delta \in \mathbb{R}$ such that $\alpha_{1}<\delta<\alpha_{2}$. We make the change of variable $s \mapsto s+\delta$ resulting into a polynomial $p_{\delta}(s)=p(s+\delta)$. The roots of $p_{\delta}$ are located at $\alpha_{k}^{\prime}=\alpha_{k}-\delta$ and it is easy to see that $\alpha_{1}^{\prime}<0$ and $\alpha_{k}^{\prime}>0$ for $k=2, \ldots, m$. Then we apply the map $s \mapsto s^{2}$ which produces a polynomial $q(s)=p_{\delta}\left(s^{2}\right)$ with a pair of pure conjugated complex roots at $\pm \mathbf{i} \sqrt{\delta-a_{1}}$ and pairs of symmetric real roots at $\pm \sqrt{a_{k}-\delta}$ for $k=2, \ldots, m$. Under the hypothesis of separability, $q(s)=q_{R}(s) q_{C}(s)$ and, undoing in reverse order the changes of variables, $s \mapsto \sqrt{s} \mapsto s+\delta$, we obtain that $q_{R}(\sqrt{s-\delta})=\left(s-\alpha_{1}\right)$ and $q_{C}(\sqrt{s-\delta})=p(s) /(s-$ $\alpha_{1}$ ). Therefore, we might isolate roots of $p$ by successively repeating the procedure onto $q_{C}$. This is a contradiction since from the Galois theory it is well known that it is not always possible to solve a polynomial equation by radicals.

\section{Conclusions}

We overcome the drawbacks of the original Schur-Cohn criterion for delay systems, namely, the high degree of the polynomial that emerges from the Schur-Cohn matrix and the difficulty of detection of complex roots, especially pure imaginary roots, due to the propagation of numerical errors. In particular, we decrease the degree of the generated polynomial to a half and we reduce the problem numerically to that of seeking the real roots of this polynomial, not the complex ones. These properties are exactly those that make the Rekasius substitution criterion so attractive in the stability theory of delay systems. Therefore, from a practical viewpoint, the modified Schur-Cohn criterion is comparable to other widely used criteria in the literature. Our method is based on making transformations on the Schur-Cohn matrix via triangular matrices over a polynomial ring in one unknown. This leads to the determinant of the Jury matrix over a polynomial ring, which is proved to be an even polynomial. The pure imaginary roots of the Schur-Cohn polynomial are those of the even polynomial, and in virtue of the change of variable $\omega \mapsto \sqrt{\omega}$, we only need to seek real roots, which is more precise from a numerical viewpoint, while the degree of the polynomial is halved.

\section{Conflict of Interests}

The author declares that there is no conflict of interests regarding the publication of this paper.

\section{References}

[1] T. Vyhlidal, J.-F. Lafay, and R. Sipahi, Delay Systems: From Theory to Numerics and Applications, Advances in Delays and Dynamics, Springer, New York, NY, USA, 2014.

[2] K. Gu, J. Chen, and V. L. Kharitonov, Stability of Time-Delay Systems, Birkhaauser, Boston, Mass, USA, 2003.
[3] C. Bonnet, A. R. Fioravanti, and J. R. Partington, "Stability of neutral systems with commensurate delays and poles asymptotic to the imaginary axis," SIAM Journal on Control and Optimization, vol. 49, no. 2, pp. 498-516, 2011.

[4] H. Fazelinia, R. Sipahi, and N. Olgac, "Stability robustness analysis of multiple time-delayed systems using "building block" concept," IEEE Transactions on Automatic Control, vol. 52, no. 5, pp. 799-810, 2007.

[5] A. R. Fioravanti, C. Bonnet, and H. Ozbay, "Stability of fractional neutral systems with multiple delays and poles asymptotic to the imaginary axis," in Proceedings of the 49th IEEE Conference on Decision and Control, pp. 31-35, Atlanta, Ga, USA, December 2010.

[6] A. R. Fioravanti, C. Bonnet, H. Özbay, and S.-I. Niculescu, "A numerical method to find stability windows and unstable poles for linear neutral time-delay systems," in Proceedings of the 9th IFAC Workshop on Time Delay Systems (TDS '10), pp. 183-188, Prague, Czech Republic, June 2010.

[7] A. R. Fioravanti, C. Bonnet, H. Özbay, and S.-I. Niculescu, "A numerical method for stability windows and unstable rootlocus calculation for linear fractional time-delay systems," Automatica, vol. 48, no. 11, pp. 2824-2830, 2012.

[8] N. Olgac and R. Sipahi, "A practical method for analyzing the stability of neutral type LTI-time delayed systems," Automatica, vol. 40, no. 5, pp. 847-853, 2004.

[9] R. Sipahi, "Delay-margin design for the general class of singledelay retarded-type LTI systems," International Journal of Dynamics and Control, vol. 2, no. 2, pp. 198-209, 2014.

[10] S. Barnett, Polynomials and Linear Control Systems, Marcel Dekker, New York, NY, USA, 1983.

[11] J. Chen, G. Gu, and C. N. Nett, "A new method for computing delay margins for stability of linear delay systems," Systems and Control Letters, vol. 26, no. 2, pp. 107-117, 1995.

[12] P. Fu, S.-I. Niculescu, and J. Chen, "Stability of linear neutral time-delay systems: exact conditions via matrix pencil solutions," IEEE Transactions on Automatic Control, vol. 51, no. 6, pp. 1063-1069, 2006.

[13] K. Walton and J. E. Marshall, "Direct Method for TDS Stability Analysis," IEE Proceedings D: Control Theory and Applications, vol. 134, no. 2, pp. 101-107, 1987.

[14] J. H. Su, "The asymptotic stability of linear autonomous systems with commensurate time delays," IEEE Transactions on Automatic Control, vol. 40, no. 6, pp. 1114-1117, 1995.

[15] J. Louisell, "A matrix method for determining the imaginary axis eigenvalues of a delay system," IEEE Transactions on Automatic Control, vol. 46, no. 12, pp. 2008-2012, 2001.

[16] N. Olgac and R. Sipahi, "An exact method for the stability analysis of time-delayed linear time-invariant (LTI) systems," IEEE Transactions on Automatic Control, vol. 47, no. 5, pp. 793797, 2002.

[17] Z. V. Rekasius, "A stability test for systems with delays," in Proceedings of the Joint Automatic Control Conference, 1980, paper no. TP9-A.

[18] A. Thowsen, "The Routh-Hurwitz method for stability determination of linear differential-difference systems," International Journal of Control, vol. 33, no. 5, pp. 991-995, 1981.

[19] R. Sipahi and N. Olgac, "A comparative survey in determining the imaginary characteristic roots of LTI time delayed systems," in Proceedings of the 16th Triennial World Congress of International Federation of Automatic Control (IFAC '05), vol. 16, pp. 390-399, Prague, Czech Republic, July 2005. 
[20] D. Hinrichsen and A. J. Pritchard, Mathematical Systems Theory I, vol. 48 of Texts in Applied Mathematics, Springer, Berlin, Germany, 2005.

[21] H. Gluesing-Luerssen, Linear Delay-Differential Systems with Commensurate Delays: An Algebraic Approach, vol. 1770 of Lecture Notes in Mathematics, Springer, 2002. 


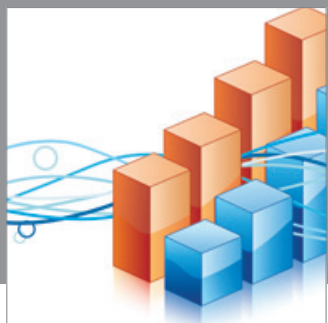

Advances in

Operations Research

mansans

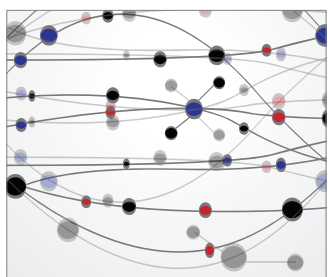

The Scientific World Journal
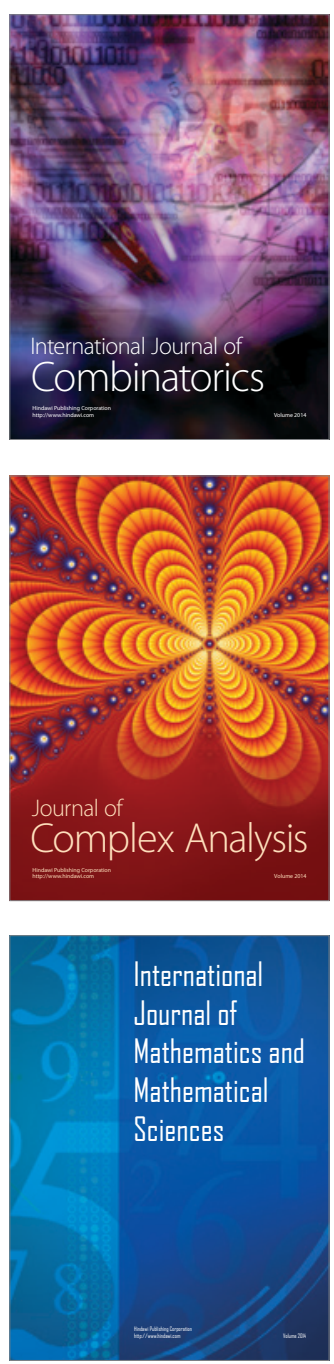
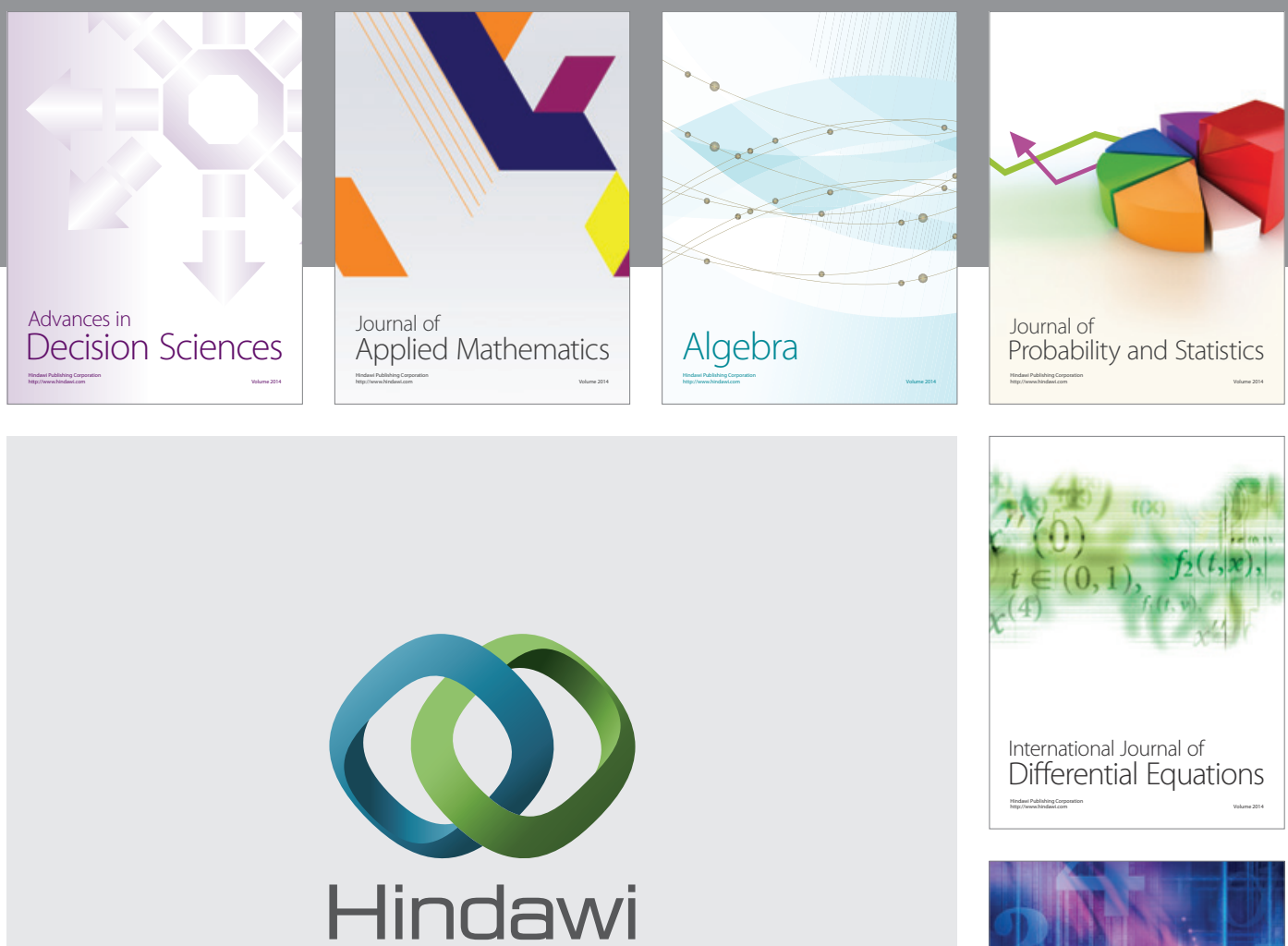

Submit your manuscripts at http://www.hindawi.com
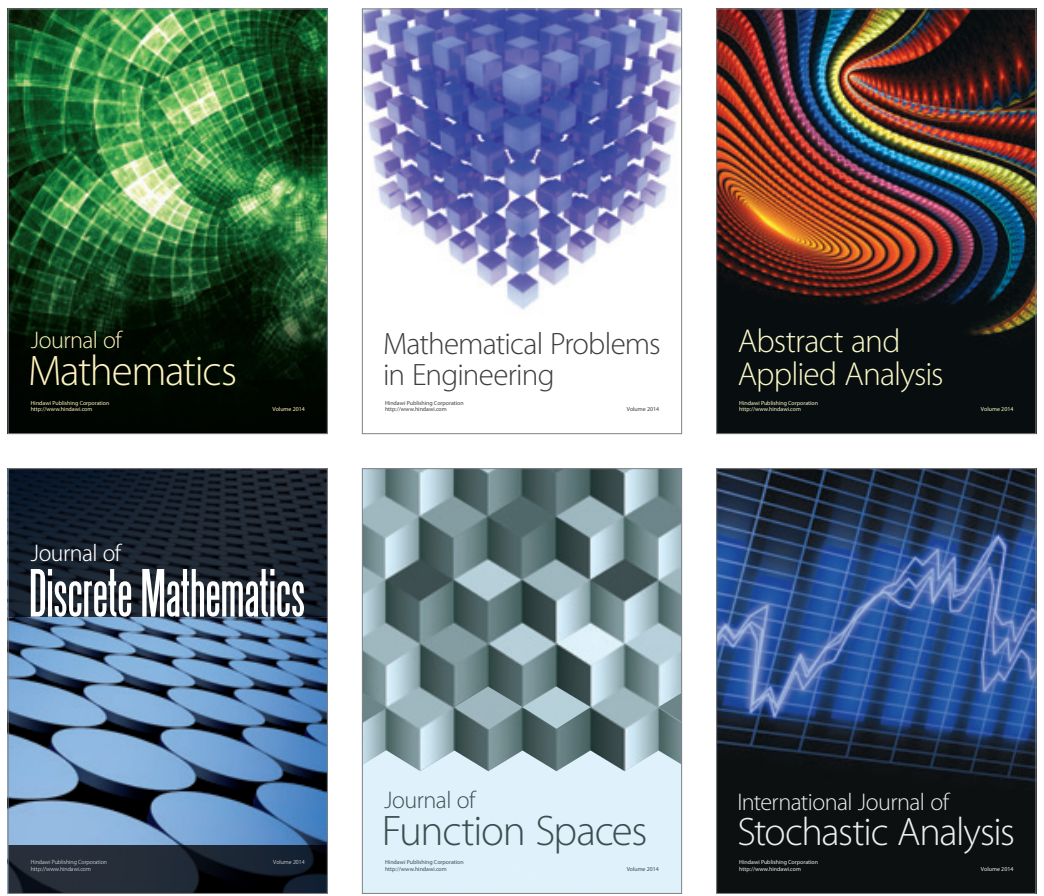

Journal of

Function Spaces

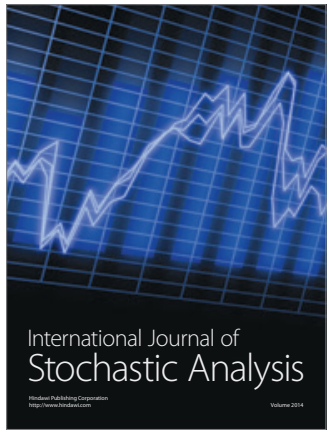

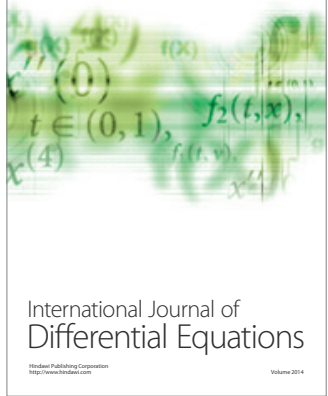
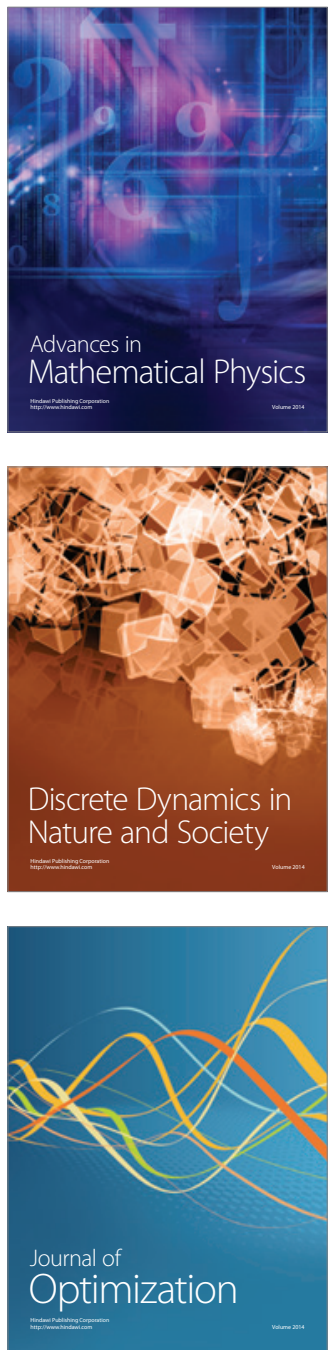